\title{
Trouble Spots in Online Direct-to-Consumer Prescription Drug Promotion: Teaching Drug Marketers How to Inform Better or Spin Better?
}

\author{
Comment on "Trouble Spots in Online Direct-to-Consumer Prescription Drug Promotion: A \\ Content Analysis of FDA Warning Letters"
}

\section{Evan Doran*}

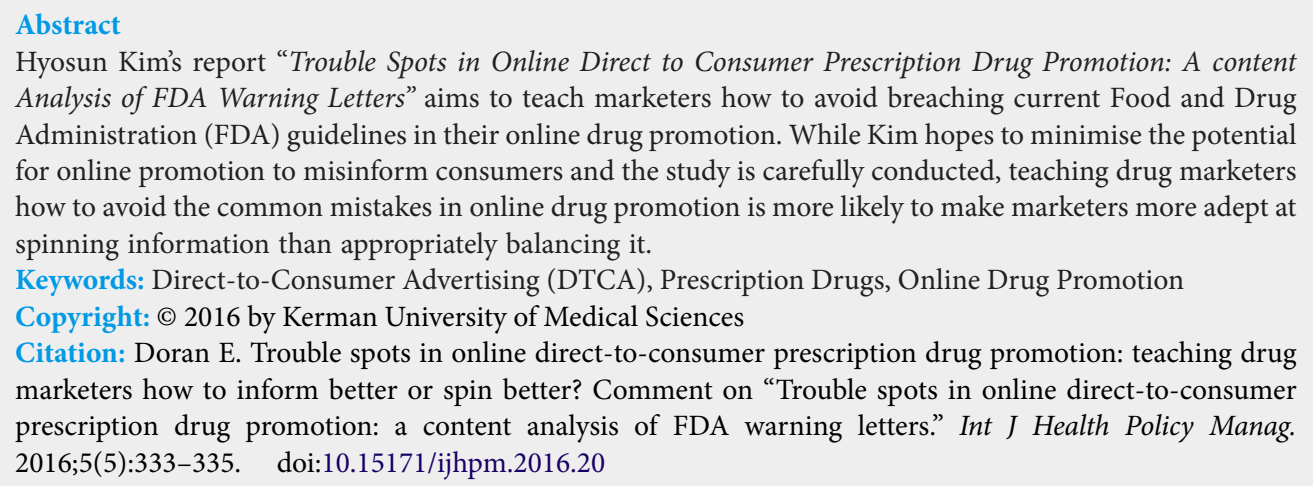

\section{Article History:}

Received: 15 December 2015 Accepted: 19 February 2016 ePublished: 21 February 2016

\author{
*Correspondence to: \\ Evan Doran \\ Email: Evan.doran@newcastle.edu.au
}

$\mathrm{D}$ irect-to-consumer advertising (DTCA) of prescription pharmaceuticals, whether the medium is print, broadcast or online, is a vexed issue. Discussion of the pros and cons of prescription drug DTCA is always morally charged with valid claims and equally valid counter-claims, plausible allegations and cogent rebuttals, heroic narratives of consumer empowerment and atrocity stories of therapeutic disasters. If we pose the bald question "is DTCA helpful" the best one can answer on the available evidence is a frustrating: 'yes and no, maybe, it depends.' Understandably, many policy-makers will conclude that any 'truth' to be found on the question of whether DTCA is 'therapeutic or toxic?' is hopelessly relative to an individual's ideological commitments. It would be hoping for too much to expect that Hyosun Kim's report "Trouble Spots in Online Direct to Consumer Prescription Drug Promotion: A content Analysis of FDA Warning Letters" could do anything close to resolving the tricky substantive issues it raises. However, to be fair, it does reinforce the notion that without restraint online prescription drug promotion may be as problematic as it is productive.

Kim's research report enters this murky fray to offer drug marketers a way to optimise the likelihood of their online advertising being helpful, or at least not misleading. There is much to commend it. It is a sound piece of descriptive qualitative research and the author should be applauded for seeking to improve the quality online DTCA (or e-DTCA) and for undertaking a rigorous study to do so. Kim identifies a pressing issue for those concerned with quality (ie, appropriate, timely, and safe) use of prescription medicines
- the quality of medicine information rebounding through the online environment ie, the internet and social media. Unquestionably, the ubiquity of the internet and more recently social media have changed the way people seek and receive health information and pharmaceutical marketers have been quick to exploit this potential. The rapidity of this change has given rise to concerns that policy and regulation have not kept pace and that controls applied to DTCA in traditional media are not adequate for the very different communicative environment and 'participatory culture' of the internet and social media. Food and Drug Administration (FDA) guidelines have been developed but are somewhat ad hoc.

Unsurprising then that marketers and regulators may find themselves' occasionally at odds over certain elements of online drug campaigns. Kim sees a way to address this - teach marketers how to avoid breaching current FDA guidelines in a way that prompts the FDA to issue a 'notice of violation' (NOV) warning letter. The aim is 'diagnostic', to qualitatively study such letters and identify how a particular advertising campaign or part thereof has breached FDA guidelines and thereby provide drug marketers with the 'context' to the FDA's issuing a NOV.

Kim's study asks a set of straightforward descriptive research questions: What types of online promotional received the most letters? What drug categories that have received letters? What types of legal violations does the FDA allege? Do alleged violations differ by online promotion type? The methods used to answer these questions are also relatively straightforward, appropriate and carefully conducted. The letters have been 
subject to a content analysis ie, they have been read, coded and organised into descriptive categories relevant to each research question. The analysis showed that: the majority of violations were related to advertisements on branded drug websites, online paid advertisements and online video promotion; the promotion of drugs for chronic diseases attracted most warning letters; inadequate risk and efficacy information were the most frequently cited violations; and online advertisements were the type of promotion most frequently subject to a warning letter. Kim's most significant finding is that online prescription drug advertising, like DTCA in broadcast media, fails to present risk and benefit information in a balanced manner. The main recommendation of the paper is for the FDA to provide "comprehensive guidelines that address the accessibility and visibility of information would help pharmaceutical marketers avoid putting so many misleading promotional activities online."

It is clear that Kim accepts the proposition that online DTCA, properly guided and responsibly executed, is a force for good. Before I challenge this proposition, I declare that I have a track record in opposing DTCA in my home country of Australia having researched and published on the issue (eg, Hogue et al, ${ }^{3}$ Doran and Lofgren ${ }^{4}$ ). My partisanship on this issue, however, does not extend to denying that there are considerable challenges in making a morally or empirically watertight case against DTCA. Both advocates and opponents of DTCA may, respectively, muster an inventory of benefits for or litany of complaints against DTCA and offer theoretical and empirical support for its benefits and drawbacks. To be fair, both sides can offer cogent arguments. The claims and counterclaims are well-rehearsed in the literature and readers may refer to the following references for more detailed descriptions of the opposing arguments..$^{5-9}$ Briefly the purported benefits include, redressing under-diagnosis and under-treatment of conditions such as diabetes and depression, improving patient compliance with treatment, improving patient education and improving patient-doctor interaction. The purported harms include, misinforming patients, inappropriate prescribing, prompting rapid uptake of unproven medicines and increasing drug expenditure. There is not the space here to trenchantly pull apart the opposing positions and the evidence that is mobilised in support of their arguments. In this commentary, I will offer a counterpoint to Kim's main argument that with better understanding of 'context' and stronger guidelines drug marketer's will able and willing to avoid presenting information in a misleading way, and thereby help consumers have better discussions with their doctor and make better treatment decisions.

The context Kim refers to is essentially the detail of the FDA's judgement that the product information presented within online drug promotion does not adequately balance risks and benefits. The solution it appears is to institute more comprehensive guidelines that will help marketers strike a balance - give equal space and draw equal attention to information on risks as well as benefits. While an understanding of context, as defined by Kim, may prompt marketers to give greater attention to the balance of the drug information it is far from obvious that marketers will forego framing their communication in a way that persuades consumers that the benefits are likely and the risks remote. This proposal seriously underplays drug marketers' main motivation. The provision of information about pharmaceutical products by marketers is from the very start a fraught exercise by virtue of the incontrovertible fact that drug marketing is predicated on persuasion to maximise sales and return to shareholders. Balancing benefit-risk information is always subordinate to these imperatives. As I have argued elsewhere if maximising sales is a perfectly legitimate objective, it is also indiscriminate - with the capacity to consume as important as the capacity to benefit. Secondly, it is difficult in the extreme to imagine guidelines that can adequately counter drug marketers' essential urge to 'spin' product information to emphasise the potential benefits and underplay the potential risks or uncertain efficacy. As McKenzie et $\mathrm{al}^{10}$ have pointed out, there are no simple evaluative principles available by which the quality of drug product information may be objectively judged. Drug information may be factually accurate but its presentation involves the use of evocative imagery and language that (subtly or otherwise) convey narratives and themes that move beyond facts to appeal to universals such as the dread of sickness and desire to be better. Compelling marketers to ensure information is balanced visible and accessible does not address these more potent elements of advertising.

Kim asserts that "DTCA helps consumers have better discussions with their physicians and to make better healthrelated decisions as active consumers." Here we can see the now familiar trope of the empowered consumer that has been mobilised by pharmaceutical companies since they first commenced targeting the public with DTCA in the 1990s. But if it is plausible that e-DTCA gives consumers the information they need to actively engage with their doctors in treatment decisions, it is equally plausible that this engagement will follow a 'script' that favours the branded drug.

Mogull and Balzhiser's ${ }^{11}$ rhetorical analysis of web-based DTCA for prescription drugs to treat migraine shows how the doctor-patient dialogue can be shaped toward diagnosis and the issuing of a prescription. The online promotional materials employed imagery and text that emphasise the capacity of the consumer to take control of their illness, primarily by talking to their doctor about the branded drug. The promotional materials analysed by Mogull and Balzhiser do more than merely prompt the reader to 'ask your doctor', they also provide a set of questions that the consumer may use to guide the discussion ie, the consumer arrives at their doctors office with a prepared script. Under such circumstances, the most influential presence in the ensuing discussion is neither the doctor nor the patient but the pharmaceutical company. The provision of information here is less about empowerment and more about direction.

Although laudable, Kim's aim of teaching drug marketers how to avoid the common mistakes in online drug promotion is more likely to make marketers more adept at spinning information than appropriately balancing it. Nevertheless, given that DTCA is legally permissible in the United States and it is unlikely that this will be reversed, Kim's recommendation for more comprehensive guidelines for online prescription drug promotion needs to be heeded. The increasing pervasiveness of digital communication will mean 
drug marketers will continue to adopt innovative e-DTCA tactics $^{12}$ and guidelines must be flexible enough to keep abreast of and adequately account for developments in online drug promotion. For this to be achieved, more will need to be known about how pharmaceutical companies are adapting DTCA to the online environment. As Mackey and Liang ${ }^{12}$ have noted, here lies the most urgent challenge in ensuring that DTCA is more productive than problematic, compelling pharmaceutical companies to be more transparent about what they are doing and spending when 'informing' consumers.

\section{Ethical issues}

Not applicable.

\section{Competing interests}

Author declares that he has no competing interests.

\section{Author's contribution}

$E D$ is the single author of the paper.

\section{References}

1. Ventola CL. Direct-to-consumer pharmaceutical advertising: therapeutic or toxic? P T. 2011;36(10):669-684.

2. Kim H. Trouble spots in online direct-to-consumer prescription drug promotion: a content analysis of FDA warning letters. Int J Health Policy Manag. 2015;4(12):813-821. doi:10.15171/ ijhpm.2015.157

3. Hogue MC, Doran E, Henry DA. A prompt to the web: the media and health information seeking behaviour. PloS One. 2012;7(4):e34314. doi:10.1371/journal.pone.0034314

4. Doran E, Löfgren H. Drug promotion in Australia: policy contestation and the tightening of regulation. Australian Review of Public Affairs. 2013;11(2):19-41.

5. Atherly $\mathrm{A}$, Rubin $\mathrm{PH}$. The cost-effectiveness of direct-toconsumer advertising for prescription drugs. Med Care Res Rev. 2009;66(6):639-657. doi:10.1177/1077558709335362

6. Harker M, Harker D. Direct-to-consumer advertising of prescription medicines: A systematic review of the evidence from the perspective of the consumer. Journal of Medical Marketing: Device, Diagnostic and Pharmaceutical Marketing. 2007;7(1):4554. doi:10.1057/palgrave.jmm.5050060

7. Auton $F$. The advertising of pharmaceuticals direct to consumers: A critical review of the literature and debate. International Journal of Advertising. 2004;23(1):23-52. doi:10.1080/02650487.2004.1 1072871

8. Auton F. Direct-to-consumer-advertising (DTCA) of pharmaceuticals: An updated review of the literature and debate since 2003. Economic Affairs. 2006;26(3):24-32. doi:10.1111/ j.1468-0270.2006.00646.x

9. Rod M, Saunders $S$. The informative and persuasive components of pharmaceutical promotion - An argument for why the two can coexist. International Journal of Advertising: The Review of Marketing Communications. 2009;28(2):313-349. doi:10.2501/ S0265048709200558

10. Mackenzie FJ, Jordens CF, Ankeny RA, McPhee J, Kerridge IH. Direct-to-consumer advertising under the radar: the need for realistic drugs policy in Australia. Intern Med J. 2007;37(4):224228.

11. Mogull SA, Balzhiser D. Pharmaceutical companies are writing the script for health consumerism. Communication Design Quarterly Review. 2015;3(4):35-49.

12. Mackey TK, Liang BA. It's time to shine the light on directto-consumer advertising. Ann Fam Med. 2015;13(1):82-85. doi:10.1370/afm.1711 